\title{
Dynamic Relationship Between Government Spending, Final Consumption and Savings: Evidence from Southeast Europe
}

Submitted 25/02/20, 1st revision 21/03/20, 2nd revision 15/04/20, accepted 20/05/20

\begin{abstract}
Nakije Kida ${ }^{1}$
Abstarct:

Purpose: The purpose of this study is to explore the dynamic relationships between government saving, family consumption, private savings and population in selected countries in Southeast Europe.

Design/Methodology/Approach: The descriptive statistical analysis was used to answer the research question, using Pearson correlation analysis, Unit root test, ARDL cointegration test, Johansen Tests for Cointegration and Granger Causality test. The data set covers the the period 2004-2018 in US\$, calculated per capita.

Findings: The results find a statistically significant and positive long-term relationship (lag_lag) between family consumption $\left(G_{t}\right)$, government saving $\left(C_{t}\right)$, gross savings $\left(G S_{t}\right)$ and per capita income $\left(Y_{t}\right)$. Though, the relationship between family consumption and population is considered statistically significant but negative. However, in the short term there is no causal link in any of the variables.

Practical Implications: The results are clear and a strong message for policymakers and decision makers to prioritize increased government spending that causes final consumption growth as a catalyst to increase the demand for goods and services.

Originality: This research paper highlights an empirical analysis based on real data obtained from the World Bank Statistics for five countries in the region.
\end{abstract}

Keywords: Family consumption, government spending, gross savings, per capita income, Granger causality.

JEL Codes: E2, E6, E21, H5, H31.

Paper type : Research article.

${ }^{1}$ Faculty of Economic Sciences, College AAB, e-mail: nakije.kida@ universitetiaab.com; 


\section{Introduction}

The study analyzes dynamics of the relationship between spending consumption $(\mathrm{Ct})$, government spending (Gt) and Gross Savings ( GS) as important factors in the economies of Kosovo, Albania, Northern Macedonia, Croatia and Serbia.

The basic question that arises is:

Are there positive short-term or long-term relationships between final consumption and government spending in Kosovo, Albania, Montenegro, Serbia and Northern Macedonia?

It should be known whether government spending complements or replaces or has nothing to do with final consumption for the period 2004-2018 in five SEE countries. Ramey and Shapiro (1998), used narrative approaches, and concluded that government consumption causes "crowding-out" of the private consumption, quoted by Ercolani (2007, pp. 1-3).

Whereas, if we start from the point of view of the standard neoclassical as well as the New-Keynesian theory, the authors Baxter and King (1993) predict that private consumption falls as a result of the positive shock of government spending. Linnemann and Schabert (2003) formulate a New-Keynesian model, who state that government spending is positively related to private consumption, while Gali, Lupez-Salido and Valles (2007), present the imperfection of the market, where a part of the population cannot borrow or lend (quoted by Ercolani, 2007, p. 3).

Blanchard and Perotti (2002), Canzoneri et al. (2002), Fatas and Mihov (2002) also point out that shocks in government spending appear to be associated with increased private consumption.

\section{Literature Review}

The relationship between family consumption and government spending still remains a topic of discussion in many academic and political debates, as it is a very important indicator of the formulation of fiscal policies in the country.

Keynes (1936), introduced the concepts of consumption function, the principle of effective demand and liquidity preference, and gave new priority to multiplier and marginal capital efficiency (Chapsa et al., 2018). Keynesian theory predicts an increase in private consumption in response to the shock of government spending (Keynes, 1936). Keynes's influence in the 1970s was due to stagnation, and this skepticism was strongly lost in the 2008 crisis, reviving interest in Keynesian economy. 
Analyzed the importance of savings in the economic development (Komayjani and Rahmani, 1993) each country needs investment to achieve economic growth; the necessary condition for investment is savings (Mojtahed and Karami, 2003). The savings variable was also analyzed by Bebezuk and Musalem (2006), based on a sample of 48 developed and developing countries over the period 1980- 2004 using panel data techniques to conclude that dependency rates in old age and urbanization rates have negative correlation with savings.

Low savings hinder economic growth (Karami, 2008: 249). National savings for the sample countries (Kosovo, Albania, Montenegro, Northern Macedonia and Serbia) are a problem that affects the low level of investment and GDP growth per capita. In their study, Bebezuk and Musalem (2006), processed during the period 1980-2004, concluded that the old age of population has a negative relationship with savings, while the rate of GDP growth and trade have a positive impact on national savings.

In the research of Mojtahed and Karami (2003), economic growth and per capita income have positive effects on saving rates. While Sadi (2006) showed that not only GDP growth and current accounts affect savings but also the population growth does. The mechanisms of these effects are described in details in Aiyagari, Christiano, and Eichenbaum (1990), Baxter and King (1993), Christiano and Eichenbaum (1992), and Fatás and Mihov (2001), according to which an increase in government spending is reflected in a decrease in consumption, increase in employment and at the same time an increase in production. The multiplying action of these factors increases the return to capital and encourages the investment growth again (Galí, López-Salido and Vallés, 2004).

In conclusion, it is obvious that empirical findings on the relationship between private consumption and the increase in government spending, savings, GDP growth per capita and population vary depending on the methodology used.

\section{Methodology and Model Specification}

Our goal is to test the dynamic relationship between government consumption and family final consumption, real per capita income, gross savings and population growth in five countries in the region over a period of 15 years. The sample consists of five countries (Kosovo, Albania, Croatia, Macedonia and Serbia), while the study period covers the years 2004 to 2018, the data were collected by WDI and calculated per capita. All variables are calculated per capita.Variables have been described as:

Dependent Variable - Family Consumption $\left(\ln \mathrm{C}^{\mathrm{t}}\right)$.

Independent Variables - Government Consumption $\left(\mathrm{InG}^{\mathrm{t}}\right)$, the effect on final family consumption.

Through equation (1) (Keho, 2019), we first evaluate the theoretical model and then add other independent variables in equation (2): 
$\ln \mathrm{C}_{\mathrm{t}}=\alpha_{1}+\beta_{1} \ln \mathrm{G}_{\mathrm{t}}+\mu_{\mathrm{lt}}$

where: $\mathrm{C}_{\mathrm{t}}=$ Real private family consumption $\left(\ln \mathrm{C}^{\mathrm{t}}\right)$ dependent;

$\mathrm{G}_{\mathrm{t}}=$ is Government consumption per capita, independent;

$\mu_{1 t}$, is a term of errors that is assumed to follow a normal distribution.

Equations 2, 3 and 4, are complemented by the coefficients of the variables: Government spending $\mathrm{G}_{\mathrm{t}}$ and Final Family Consumption $\mathrm{C}_{\mathrm{t}}$, real per capita income $\left(\gamma \ln Y_{t}\right)$, Gross family savings (InGrowsSpc $\left.c_{t}\right)$ and the population $\mathrm{PT}_{\mathrm{t}}$. To come to a more convincing result, in terms of the relationship between family consumption and government spending, the increase in GDP per capita has been included as a variable (Yt) similar to other studies (Graham, 1993; Ho, 2001; Keho, 2019). Therefore, we estimate the model specified as follows:

$\ln \mathrm{C}_{\mathrm{t}}=\alpha_{2}+\beta_{2} \ln \mathrm{G}_{\mathrm{t}}+\gamma \ln \mathrm{Y}_{\mathrm{t}}+\mu_{2 \mathrm{t}}$

where, $\mathrm{Y}^{\mathrm{t}}$ is the real per capita income. The income ratio in the research question is predicted to be positive, negative or insignificant. A family consumption coefficient is assumed to be positive, negative, complementary, substituted but not insignificant in government spending and other explanatory variables (per capita income, savings and population growth rate).

In the following, we present the additional equations (3 and 4) since in the initial model we have added two control variables, gross per capita savings (InGS) and total country's population (InPT) in order to measure their effect on the two dependent variables (inCt and $\mathrm{InGt}$ ).

$\operatorname{lnCt}=\alpha_{2}+\beta_{2} \ln G_{t}+\gamma \ln Y_{t}+$ InGrowsSpc $_{t}, \operatorname{InPT}^{t}+\mu_{3 t}$

$\operatorname{lnGt}=\alpha_{2}+\beta_{2} \ln C_{t}+\gamma \ln Y_{t}+\operatorname{InGrowsSpc}_{t}, \operatorname{InPT}_{t}+\mu_{4 t}$

where: $\mathrm{InC}_{\mathrm{t}}=$ Real private family consumption;

$\ln \mathrm{G}_{\mathrm{t}}=$ Government consumption per capita;

$\mathrm{Y}_{\mathrm{t}}=$ Real Income per capita;

Grows Spc $_{\mathrm{t}}=$ Gross saving per capita (GS);

$\mathrm{InPT}_{\mathrm{t}}=$ Total population of the country, independent;

$\mu_{1 t}$ is a term of errors that is supposed to follow a normal distribution.

In many studies the real income ratio per capita $\mathrm{Y}_{t}$, is presented as positive or negative and often lower than the government consumption coefficient $\mathrm{C}^{\mathrm{t}}$. This is unclear for government consumption because it represents a complementary (substitute) relationship between government spending $\left(\mathrm{G}_{\mathrm{t}}\right)$ and private family consumption $\left(\mathrm{C}_{\mathrm{t}}\right)$. 
Whereas, real gross savings per capita (GrowsSpc $)$, may be in negative correlation with saving and government spending. What correlation has all the variables with the total population remains to be seen in the meantime during testing. It also remains to be seen, what can be the dynamic relationship with the 3 analog variables (Keho, 2019 p. 198) and 2 additional variables, i.e., a total of 5 variables, with data for Kosovo, Albania, Montenegro, Croatia and Serbia.

The research methodology continues with testing the stationarity of variables, using the longest time frame to measure the long-term effects of independent variables on the dependent variable through the ARDL test (Pesaran et al., 2001). ARDL testing method of borders (Agibaeva, 2015), used in the study is based on equation (3), the ARDL border testing method in integration is based on the author Keho (2019), as well as on other studies (Agibaeva, 2015).

$$
\begin{gathered}
\Delta \ln C_{\mathrm{t}}=\theta_{0}+\theta_{1} \ln C_{\mathrm{t}-1}+\theta_{2} \ln G_{\mathrm{t}-1+} \theta_{\mathrm{a}} \ln Y_{\mathrm{t}-1}+\sum_{\mathrm{i}=1}^{\mathrm{m} 1} Y_{1 \mathrm{i}} \Delta \ln C_{\mathrm{t}-\mathrm{i}}+\sum_{\mathrm{i}=0}^{\mathrm{m} 2} Y_{2 \mathrm{i}} \Delta \ln G_{\mathrm{t}-\mathrm{i}} \\
+\mu 1 \mathrm{t} \text {.. equ. (5) }
\end{gathered}
$$

Test statistics (F) are used to investigate the long-term relationship between variables. Only 1 and 2 year time delays are used, verifying the Hypothesis $\mathrm{H}_{0}$ : $\theta_{1}=\theta_{2}=0$ using the Akaike information criterion (AIC).

The model will be tested by diagnostic tests that are: Correlation for cross-time series for five countries, causality between variables, carrying out -ECM-based Granger causality test, based on equation (4) (Keho, 2019) and modified with additional variables in this study.

$$
\begin{aligned}
{\left[\begin{array}{lll}
\Delta & \ln & C_{\mathrm{t}} \\
\Delta & \ln & G_{\mathrm{t}} \\
\Delta & \ln & Y_{\mathrm{t}}
\end{array}\right]=} & {\left[\begin{array}{l}
a_{1} \\
a_{2} \\
a_{\mathrm{a}}
\end{array}\right]+\sum_{\mathrm{i}=1}^{p}\left[\begin{array}{lll}
\beta_{1 \mathrm{i}} & Y_{1 \mathrm{i}} & \delta_{1 \mathrm{i}} \\
\beta_{2 \mathrm{i}} & Y_{2 \mathrm{i}} & \delta_{2 \mathrm{i}} \\
\beta_{\mathrm{ai}} & Y_{\mathrm{ai}} & \delta_{\mathrm{ai}}
\end{array}\right] \times\left[\begin{array}{lll}
\Delta & \ln & C_{\mathrm{t}-\mathrm{i}} \\
\Delta & \ln & G_{\mathrm{t}-\mathrm{i}} \\
\Delta & \ln & Y_{\mathrm{t}-\mathrm{i}}
\end{array}\right]+\left[\begin{array}{l}
\lambda_{1} \\
\lambda_{2} \\
\lambda_{\mathrm{a}}
\end{array}\right] E C T_{\mathrm{t}-1} } \\
& +\left[\begin{array}{l}
e_{1 \mathrm{t}} \\
e_{2 \mathrm{t}} \\
e_{\mathrm{at}}
\end{array}\right] \ldots \ldots(6)
\end{aligned}
$$

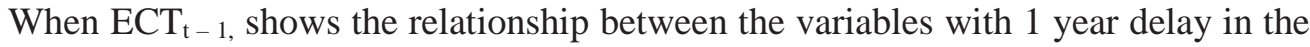
long-term plan created. Also, AIC will be used to select the optimal length of time delay. The advantage of this specification is that it can identify the causality of the relationships between variables in the short and long term. The importance of the coefficients in $\mathrm{ECT}_{\mathrm{t}-1}$, in many studies shows the cause of long-term relationships, because in the short term for example, government spending in most studies has not caused any effects on private consumption. Whether there will be positive or negative relationships in the short or long term between family final consumption and government consumption remains to be seen in this study. 
The research used annual data for the years 2004-2018, all in US dollars collected from the World Development Indicators data set of World Bank and calculated per capita.

There are five variables used in the study: Family Consumption Saving per capita $\left(\left(C_{t}\right)\right.$; Government Final Consumption Saving per capita $\left(G_{t}\right)$; Gross Savings per capita. $\left(\mathrm{GS}_{\mathrm{t}}\right)$; GDP Growth per capita $\left(\mathrm{Y}_{\mathrm{t}}\right)$; Total population of the country $\left(\mathrm{PT}_{\mathrm{t}}\right)$. The data processing is done with the Stata 13 statistical program.

In Figure 1 most of the variables have evolved, thus showing an increasing trend from 2004 to 2018, except that of population which is declining.

Figure 1. General government final consumption expenditure $\left(G_{t}\right)$, Gross savings $\left(G S_{t}\right)$, GDP per capita $\left(Y_{t}\right)$, Households and NPISHs Final consumption expenditure (Gt) and Population $\left(P T_{t}\right)$, by Years (2004-2018); Source: Worked by the author

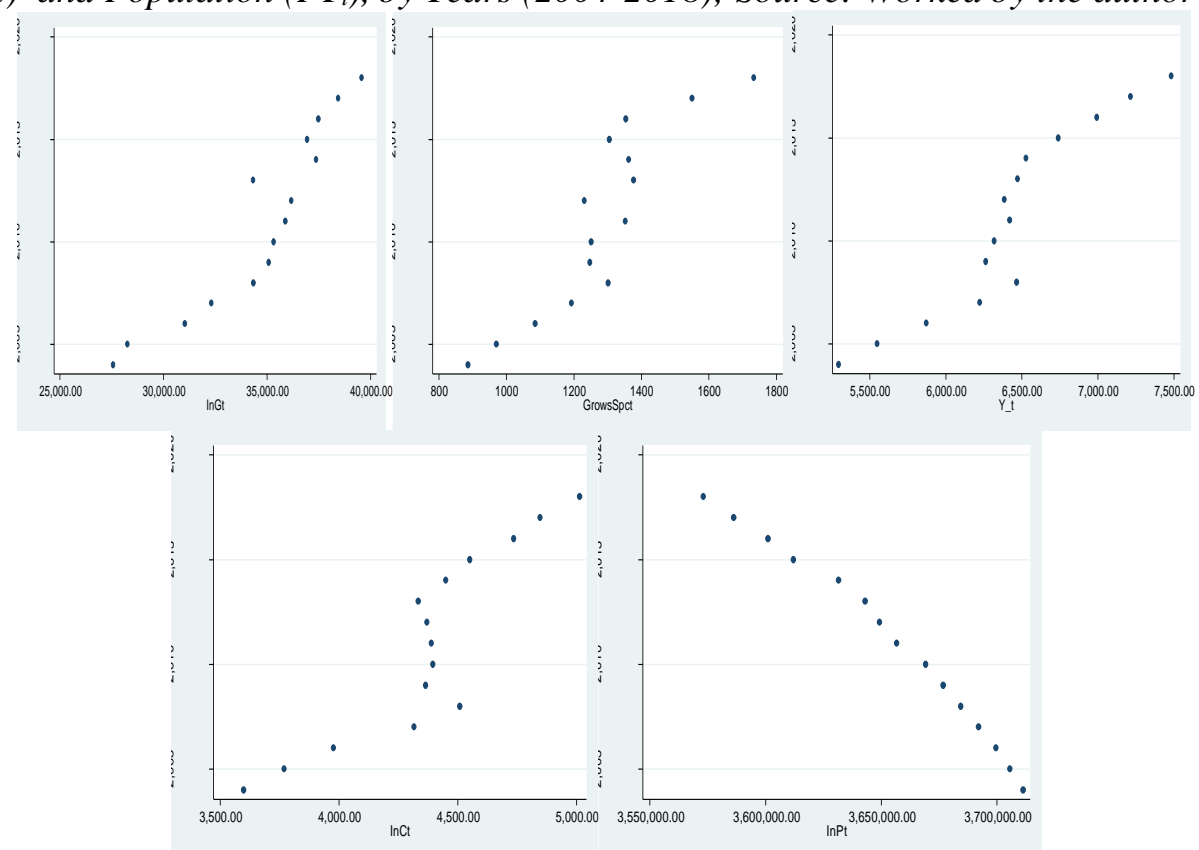

Source: Own calculations.

General government final consumption expenditure $-\mathrm{G}_{\mathrm{t}^{-}}$(constant LCU) per capita by Years - begins to grow slowly between 2004-2010, while between 2015-2018 the growth doubles.

Gross savings - $\mathrm{GS}_{\mathrm{t}}$ (per capita) by Years - from the above results we can see the increase of savings from 2010 onwards with over $25 \%$ in the following years. 
GDP per capita $-\mathrm{Y}_{\mathrm{t}^{-}} \quad$ (constant 2010 US\$) by Years - Between 2004 and 2010, the growth is distinguishable, but in the following years (2011-2018) the growth slows.

Family consumption expenditure per capita - $C_{t}-($ constant 2010 US\$) per capita by Years - have an increase between 2010-2015, while by 2018 it is a positive increase but at a slower pace.

Population, total by Years $\left(\mathrm{PT}_{\mathrm{t}}\right)$ - The decline in population starts from 2004 to 2019 , a significant and worrying decline for most of these countries.

The following Figure 2, visually represent the interrelationship between the 6 variables.

Figure 2. Clearly presents the correlation between the six study variables (1. Ingrowth saving/InCt; 2. InGrowth saving/InYt; 3. InYt/iInCt; 4. InGrowth saving/InGt; 5. InYt/InGt; 6. InGt/InCt) Source: Worked by the author
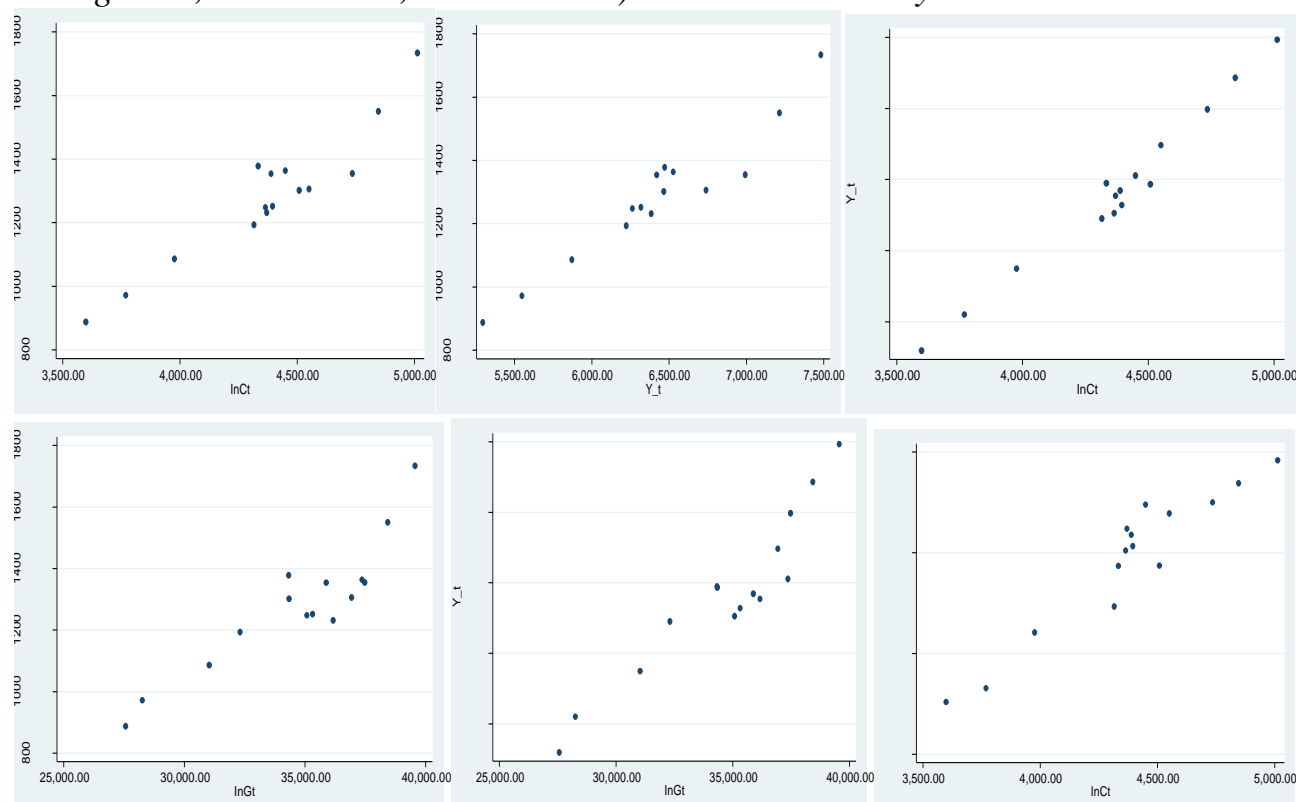

Source: Own calculations.

\section{Results}

The following analysis presents demographic data, mean value, standard deviation, minimum and maximum. All data converted per capita $(\$ 000,000)$.

First, in descriptive statistics, it is found that the value of the final consumption expenditure $(\mathrm{Ct})$ variable around the average is more concentrated because the value distribution is 8,109 units out of 2,071 average units, this distribution is the lowest. 
In addition, there is a minimum value of Government expenditure $(\mathrm{Gt})$ the value distribution is 47,430 units from the average of 1,778 . The next best variable in terms of distribution is Gross Saving (GrowsSpct) which has an average of 2,925 units with an average distribution of 52,600 units, which shows that even in this case the distribution is concentrated during our analysis period. The same conclusion is reached for the variable, GDPpc, while the population has a greater difference between countries.

Table 1. Descriptive analysis (GrowsSpct; $Y_{-} t$; InGt; InCt; InPt)

\begin{tabular}{|c|c|c|c|c|c|}
\hline Variables & Gross Saving & Y_t-GDPpc & InGt & InCt- & InPt- Population \\
\hline Minimum & 2,866 & 4,089 & 1,704 & 2,057 & 6,982 \\
\hline Maximum & 3,026 , & 4,311 & 1,845 & 2,082, & 7,463 \\
\hline Mean & 2,925 & 4,252 & 1,778 & 2,071 & 7,236 \\
\hline Std. Deviation & 52,600 & 72,407 & 47,430 & 8,109 & 157,799 \\
\hline Variance & 2,766 & 5,242 & 2,249 & 65,760 & 24,900 \\
\hline Skewness & 0.835 & -1.251 & -0.353 & -0.334 & -0.116 \\
\hline Kurtosis & -0.605 & 0.516 & -1.299 & -0.943 & -1.306 \\
\hline GrowsSpct & 1 & $0.951 * *$ & $0.910 * *$ & $0.936 * *$ & $-0.882 * *$ \\
\hline$Y_{t}$ & - & 1 & $0.937 * *$ & $0.978 * *$ & $-0.923 * *$ \\
\hline $\operatorname{InG}_{\mathrm{t}}$ & - & - & 1 & $0.931 * *$ & $-0.869 * *$ \\
\hline $\mathrm{InC}_{\mathrm{t}}$ & - & - & - & 1 & $-0.876 * *$ \\
\hline $\mathrm{InP}_{\mathrm{t}}$ & - & - & - & - & 1 \\
\hline
\end{tabular}

Note: *Correlate GrowsSpct $Y_{t} ; \operatorname{In} G_{t} ; \operatorname{InC} C_{t} ; \operatorname{InPT} T_{t} ;($ obs $=15)$. C: Statistical significance at the level of $5 \% *$ and at the level of $10 \% * *$

Source: WDI, 2019 (By the author).

Also, the correlation matrix in Table 1 shows a positive relationship between private consumption, government consumption and savings. There is also a positive correlation between per capita income and savings. While there is a negative relationship between population growth and all other study variables.

Descriptive analysis by countries [data is per capita (constant 2010 US\$), Tables 2, $3,4,5$ and 6]:

Table 2. Descriptive statistics by Gross Saving between countries (Per capita)

\begin{tabular}{|c|c|c|c|c|c|c|c|c|c|c|}
\hline & $\mathrm{N}$ & $\begin{array}{c}\text { Minim } \\
\text { um }\end{array}$ & $\begin{array}{c}\text { Maximu } \\
\mathrm{m}\end{array}$ & Mean & $\begin{array}{l}\text { Std. } \\
\text { Deviat } \\
\text { ion }\end{array}$ & Variance & \multicolumn{2}{|c|}{ Skewness } & \multicolumn{2}{|c|}{ Kurtosis } \\
\hline & $\begin{array}{l}\text { Stati } \\
\text { stic }\end{array}$ & $\begin{array}{c}\text { Statisti } \\
\mathrm{c}\end{array}$ & Statistic & Statistic & \begin{tabular}{|c} 
Statisti \\
c \\
\end{tabular} & Statistic & $\begin{array}{c}\text { Statisti } \\
\mathrm{c}\end{array}$ & $\begin{array}{l}\text { Std. } \\
\text { Error }\end{array}$ & $\begin{array}{c}\text { Statisti } \\
\mathrm{c} \\
\end{array}$ & $\begin{array}{l}\text { Std. } \\
\text { Error }\end{array}$ \\
\hline Albania & 15 & 666.6 & 911.3 & 783.9 & 73.31 & $5,375.2$ & 0.041 & 0.580 & -1.115 & 1.121 \\
\hline Croatia & 15 & 2,255 & 3,745 & 2,929 & 395 & 156,769 & 0.444 & 0.580 & 0.267 & 1.121 \\
\hline Kosovo & 15 & 432 & 1,010 & 698 & 187 & 34,985 & -.035 & 0.580 & -0.945 & 1.121 \\
\hline
\end{tabular}




\begin{tabular}{lr|r|r|r|r|r|rr|rr} 
North Macedonia & 15 & 402 & 2,002 & 1,165 & 470 & 221,533 & 0.049 & 0.580 & -0.947 & 1.121 \\
Serbia & 15 & 643 & 1,277 & 822 & 178 & 31,827 & 1.052 & 0.580 & 1.525 & 1.121 \\
Valid N (listwise) & 15 & & & & & & & & & \\
\hline
\end{tabular}

Source: Own calculations.

Regarding the above results (Table 2), we say that we do not have a similar result between countries for Gross saving per capita. Albania has minimal Gross saving per capita between 2004-2018 in the Western Balkans.

Table 3. Descriptive statistics by GDPpc per capita between countries

\begin{tabular}{|c|c|c|c|c|c|c|c|c|c|c|}
\hline & $\mathrm{N}$ & $\begin{array}{c}\text { Minimu } \\
\mathrm{m}\end{array}$ & $\begin{array}{l}\text { Maxi } \\
\text { mum }\end{array}$ & Mean & $\begin{array}{c}\text { Std. } \\
\text { Deviatio } \\
\mathrm{n}\end{array}$ & Variance & \multicolumn{2}{|c|}{ Skewness } & \multicolumn{2}{|c|}{ Kurtosis } \\
\hline & $\begin{array}{c}\text { Statisti } \\
\text { c } \\
\end{array}$ & Statistic & $\begin{array}{l}\text { Statist } \\
\text { ic }\end{array}$ & Statistic & Statistic & Statistic & $\begin{array}{c}\text { Statisti } \\
\mathrm{c} \\
\end{array}$ & $\begin{array}{l}\text { Std. } \\
\text { Error }\end{array}$ & $\begin{array}{c}\text { Statisti } \\
\mathrm{c}\end{array}$ & $\begin{array}{l}\text { Std. } \\
\text { Error }\end{array}$ \\
\hline Albania & 15 & 2,887 & 5,075 & 4,058 & 653 & 427,66 & -0.384 & 0.580 & -.705 & 1.121 \\
\hline Croatia & 15 & 12,981 & $\begin{array}{c}15,87 \\
0\end{array}$ & 14,248 & 794.70 & 631,56 & 0.599 & 0.580 & -.280 & 1.121 \\
\hline Kosovo & 15 & 2,625 & 4,193 & 3,401 & 467.99 & 219,01 & 0.072 & 0.580 & -.824 & 1.121 \\
\hline North Macedonia & 15 & 3,591 & 5,394 & 4,585 & 552.072 & 304,78 & -0.288 & 0.580 & -.743 & 1.121 \\
\hline Serbia & 15 & 4,392 & 6,880 & 5,781 & 668.79 & 447,28 & -0.643 & 0.580 & 0.389 & 1.121 \\
\hline Valid N (listwise) & 15 & & & & & & & & & \\
\hline
\end{tabular}

Source: Own calculations.

Regarding to the results above, we say that we have a similar results between countries by GDP per capita. Kosovo has the minimum GDP per capita between 2004-2018 in western Balkan .

Table 4. Descriptive statistics by Gavernment expediture between countries

\begin{tabular}{|c|c|c|c|c|c|c|c|c|c|c|}
\hline & $\mathrm{N}$ & $\begin{array}{c}\text { Minimu } \\
\mathrm{m}\end{array}$ & $\begin{array}{c}\text { Maximu } \\
\mathrm{m}\end{array}$ & Mean & $\begin{array}{c}\text { Std. } \\
\text { Devia } \\
\text { tion }\end{array}$ & Variance & \multicolumn{2}{|c|}{ Skewness } & \multicolumn{2}{|c|}{ Kurtosis } \\
\hline & $\begin{array}{l}\text { Stati } \\
\text { stic }\end{array}$ & Statistic & Statistic & Statistic & $\begin{array}{c}\text { Statist } \\
\text { ic }\end{array}$ & Statistic & $\begin{array}{c}\text { Statisti } \\
\text { c } \\
\end{array}$ & $\begin{array}{l}\text { Std. } \\
\text { Error }\end{array}$ & $\begin{array}{c}\text { Statisti } \\
\mathrm{c}\end{array}$ & $\begin{array}{l}\text { Std. } \\
\text { Error }\end{array}$ \\
\hline Albania & 15 & 29,639 & 56,562 & 44,855 & 9,189 & $\begin{array}{r}84,442,94 \\
2\end{array}$ & -.378 & 0.580 & -1.176 & 1.121 \\
\hline Croatia & 15 & 13,295 & 17,345 & 15,431 & 1,032 & $1,067,072$ & -.491 & 0.580 & 0.837 & 1.121 \\
\hline Kosovo & 15 & 297 & 480 & 372 & 70 & 4,972 & 0.842 & 0.580 & -0.956 & 1.121 \\
\hline North Macedonia & 15 & 23,995 & 31,080 & 28,158 & 2,171 & $4,716,610$ & -0.871 & 0.580 & -0.197 & 1.121 \\
\hline $\begin{array}{l}\text { Serbia } \\
\text { Valid N (listwise) }\end{array}$ & 15 & 70,454 & 93,603 & 84,578 & 6,094 & $\begin{array}{r}37,144,76 \\
0\end{array}$ & -1.389 & 0.580 & 2.002 & 1.121 \\
\hline
\end{tabular}

Source: Own calculations.

Even with the above results (table 4), we say that we do not have a similar result between countries by the General Government. Kosovo has the lowest government spending per capita, while Serbia has the highest government spending between 2004-2018 in the Western Balkans. 
Dynamic Relationship Between Government Spending, Family Consumption, and Savings: 530

Evidence from Southeast Europe

Table 5. Descriptive statistics by Final consumption expenditure between countries

\begin{tabular}{|c|c|c|c|c|c|c|c|c|c|c|}
\hline & $\mathrm{N}$ & $\begin{array}{c}\text { Minimu } \\
\mathrm{m}\end{array}$ & $\begin{array}{c}\text { Maximu } \\
\mathrm{m}\end{array}$ & Mean & $\begin{array}{c}\text { Std. } \\
\text { Deviati } \\
\text { on }\end{array}$ & Variance & \multicolumn{2}{|c|}{ Skewness } & \multicolumn{2}{|c|}{ Kurtosis } \\
\hline & $\begin{array}{l}\text { Stati } \\
\text { stic }\end{array}$ & Statistic & Statistic & Statistic & $\begin{array}{c}\text { Statisti } \\
\mathrm{c}\end{array}$ & Statistic & $\begin{array}{c}\text { Statisti } \\
\mathrm{c}\end{array}$ & $\begin{array}{c}\text { Std. } \\
\text { Erro } \\
\mathrm{r} \\
\end{array}$ & $\begin{array}{c}\text { Statisti } \\
\mathrm{c}\end{array}$ & $\begin{array}{l}\text { Std. } \\
\text { Error }\end{array}$ \\
\hline Albania & 15 & 1,888 & 3,736 & 3,050 & 588 & 346,627 & -.954 & 0.58 & -251 & 1.121 \\
\hline Croatia & 15 & 13,295 & 17,345 & 15,431 & 1,032 & $1,067,072$ & -.491 & 0.58 & 0.837 & 1.121 \\
\hline Kosovo & 15 & 2,352 & 3,731 & 2,968 & 483 & 233,848 & 0.346 & 0.58 & -151 & 1.121 \\
\hline $\begin{array}{l}\text { North } \\
\text { Macedonia }\end{array}$ & 15 & 2,746 & 3,822 . & 3,345 & 292 & 85,390 & -.329 & 0.58 & 0.034 & 1.121 \\
\hline $\begin{array}{l}\text { Serbia } \\
\text { Valid } \\
\text { (listwise) }\end{array}$ & $\begin{array}{l}15 \\
15\end{array}$ & 3,253 & 4,700 & 4,220 & 390 & 152,863 & -.635 & 0.58 & 2.271 & 1.121 \\
\hline
\end{tabular}

Source: Own calculations.

Regarding the above results (Table 5), we say that we do not have a similar result between countries for final consumption.

Albania has the lowest government spending per capita, between 2004-2018 in the Western Balkans.

Table 6. Descriptive statistics by Population in total between countries

\begin{tabular}{|c|c|c|c|c|c|c|c|c|c|c|}
\hline & $\mathrm{N}$ & Minimum & Maximum & Mean & $\begin{array}{c}\text { Std. } \\
\text { Deviatio } \\
\mathrm{n}\end{array}$ & Variance & \multicolumn{2}{|c|}{ Skewness } & \multicolumn{2}{|c|}{ Kurtosis } \\
\hline & $\begin{array}{l}\text { Stati } \\
\text { stic }\end{array}$ & Statistic & Statistic & Statistic & Statistic & Statistic & $\begin{array}{c}\text { Statisti } \\
\mathrm{c}\end{array}$ & $\begin{array}{c}\text { Std. } \\
\text { Erro } \\
\mathrm{r} \\
\end{array}$ & $\begin{array}{c}\text { Statist } \\
\text { ic }\end{array}$ & $\begin{array}{l}\text { Std. } \\
\text { Error }\end{array}$ \\
\hline Albania & 15 & $2,866,376$ & $3,026,939$ & $2,925,018$ & 52,600 & $\begin{array}{r}2,766,82 \\
7,897\end{array}$ & 0.835 & 0.58 & -605 & 1.121 \\
\hline Croatia & 15 & $4,089,400$ & $4,311,159$ & $4,252,038$ & 72,407 & $\begin{array}{r}5,242,83 \\
3,589\end{array}$ & -1.251 & 0.58 & 0.516 & 1.121 \\
\hline Kosovo & 15 & $1,704,622$ & $1,845,300$ & $1,778,931$ & 47,430 & $\begin{array}{r}2,249,60 \\
8,290\end{array}$ & -0.353 & 0.58 & -.299 & 1.121 \\
\hline $\begin{array}{l}\text { North } \\
\text { Macedoni } \\
\text { a }\end{array}$ & 15 & $2,057,048$ & $2,082,958$ & $2,071,907$ & 8,109 & $\begin{array}{r}65,760,1 \\
61\end{array}$ & -0.334 & 0.58 & -.943 & 1.121 \\
\hline Serbian & 15 & 6,98 & $7,463,157$ & $7,236,271$ & 157,799 & $\begin{array}{r}24,900,5 \\
34,416\end{array}$ & -0.116 & 0.58 & -.306 & 1.121 \\
\hline $\begin{array}{l}\text { Valid } \mathrm{N} \\
\text { (listwise) }\end{array}$ & 15 & & & & & & & & & \\
\hline
\end{tabular}

Source: Own calculations.

Regarding the above results, we say that Kosovo has the lowest population and the highest birth rate in the Western Balkans, while Serbia has the highest population but not the birth rate between 2004-2018.

Figure 3 shows the relationship between government consumption and final consumption, which shows a relative increase during the period 2004-2018.

While the correlation between gross savings and GDP per capita represents an increase between 2004 and 2018. 
Figure 3. Two way are - InGt \& InCt si dhe Two way are-Y_\& Gros Spct
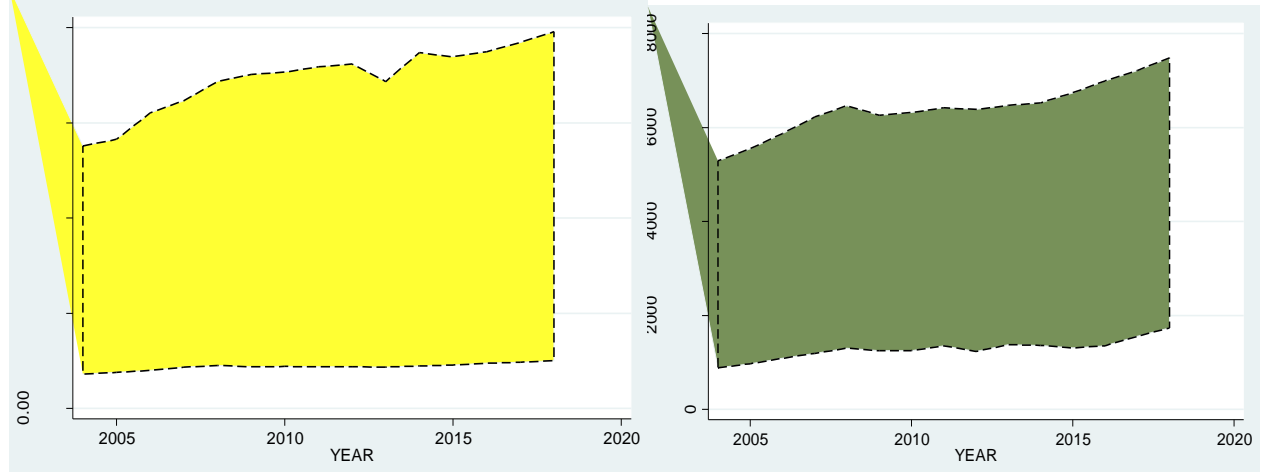

Source: Own calculations.

Unit Root test analysis (Table 7) has been done to determine whether trend data should be differentiated or regressed in time-determining functions. So, we present the existing relationships of long-term equilibrium between the variables of nonstationary time series. From the results we see that in Table 7, in Lagged difference 1 , the P-value is less than $0.5 \%$, which means that in our data in a long time an impact between the variables has been found, while the test $(t)$ is larger than 1.6 in all cases.

So we see that a period with a time lag 1 , we have the test value (T) above 1.60 in all cases, GrowsSpc $(\mathrm{t}=2.23, \mathrm{p}$ value $=.044) ; \mathrm{Y}_{\mathrm{t}}(\mathrm{t}=3.66, \mathrm{p}$ value $=.003) ; \mathrm{InG}_{\mathrm{t}}($ $\mathrm{t}=2.35, \mathrm{p}$ value $=.035) ; \operatorname{InC}_{\mathrm{t}}(\mathrm{t}=2.84, \mathrm{p}$ value $=.014)($ Table 7$):$

Table 7. Unit root test short run

\begin{tabular}{|c|c|c|c|c|c|c|c|c|c|c|}
\hline $\begin{array}{l}\text { D.Gro } \\
\text { wsSpct }\end{array}$ & Coef. & Std.Err. & $\mathrm{T}$ & $P>|t|$ & \multicolumn{2}{|c|}{ [95\% Conf.Interval } & $\mathrm{Zt}$ & $1 \%$ & $5 \%$ & $10 \%$ \\
\hline L1. & .04617 & .02070 & 2.23 & 0.044 & .00145 & .09090 & 2.23 & -2.66 & -1.95 & -1.6 \\
\hline Y_t & Coef. & Std.Err. & $\mathrm{T}$ & $\mathrm{P}>|\mathrm{t}|$ & \multicolumn{2}{|c|}{ [95\% Conf.Interval } & $\mathrm{Zt}$ & $1 \%$ & $5 \%$ & $10 \%$ \\
\hline L1. & .02411 & .00658 & 3.66 & 0.003 & .00989 & .03834 & 3.66 & -2.66 & -1.95 & -1.60 \\
\hline InGt & Coef. & Std.Err. & $\mathrm{T}$ & $\mathrm{P}>|\mathrm{t}|$ & \multicolumn{2}{|c|}{ [95\% Conf.Interval } & $\mathrm{Zt}$ & $1 \%$ & $5 \%$ & $10 \%$ \\
\hline L1. & .02328 & .00989 & 2.35 & 0.035 & .00191 & .04466 & 2.35 & -2.66 & -1.95 & -1.60 \\
\hline $\mathrm{InCt}$ & Coef. & Std.Err. & $\mathrm{T}$ & $\mathrm{P}>|\mathrm{t}|$ & \multicolumn{2}{|c|}{ [95\% Conf.Interval } & $\mathrm{Zt}$ & $1 \%$ & $5 \%$ & $10 \%$ \\
\hline L1. & .02242 & .00788 & 2.84 & 0.014 & .00539 & .03945 & 2.84 & -2.66 & -1.95 & -1.60 \\
\hline
\end{tabular}

Note: C: Family consumption expenditure per capita, Y: Percentage of GDP growth per capita, G: government final consumption expenditure per capita. GS: Gross savings per capita. * (**) denotes the rejection of the null hypothesis at the 5\% (10\%) level.

Source: Own calculations.

Now, we can proceed to the application of the ARDL (Autoregressive distributed $\mathrm{lag}^{2}$ ) bounds test to check if there is a long-run cointegration relationship among

\footnotetext{
${ }^{2}$ Authors, Pesaran, M. H. and Y. Shin, (1999:1.) point out that a large number of alternative estimates and hypothesis testing procedures have been developed specifically for analysis of the variable I (1).
} 
private consumption, government spending, growth saving and GDP growth rate per capita $\left(Y_{t}\right)$. ARDL analysis was performed with time delay (to see time differences) from, lag 5, lag 4 and lag 2. In Table 8 we see that we have significant statistical differences p- value in lag1_lag2 $=.000$, AIC $=50.9458$, and in lag2 p- value $=.000$, AIC $=50.9397^{*}$. For the longer term, Table 9 with Lag _Order 4; Table 10 with Lag _Order 2; and Table 11. Whereas, the summary data of the ARDL _lag1 test are in Table 12.

Table 8. Lag_order 1. ARDL cointegration test

\begin{tabular}{|c|c|c|c|c|c|c|c|c|}
\hline \multicolumn{8}{|c|}{.varsoc GrowsSpct Y_t InGt InCt, maxlag (5) lutstats } \\
Selection-order criteria (lutstat) \\
\hline Lag & LL & LR & df & p & FPE & AIC & HQIC & SBIC \\
\hline 0 & -347.008 & & & & $3.3 \mathrm{e}+18$ & 54.0013 & 53.9656 & 54.1751 \\
\hline 1 & -311.147 & 71.722 & 16 & 0.000 & $1.9 \mathrm{e}+17^{*}$ & 50.9458 & 50.7671 & $51.8149^{*}$ \\
\hline 2 & -295.108 & $32.079 *$ & 16 & 0.010 & $5.6 \mathrm{e}-17$ & $50.9397 *$ & $50.6181^{*}$ & 52.5041 \\
\hline
\end{tabular}

Note: Endogenous: GrowsSpct, Y_t, InGt, InCt. Exogenous:_cons

Source: Own calculations.

Table 9. Lag_Order 4. ARDL cointegration test-

\begin{tabular}{|c|c|c|c|c|c|c|c|c|}
\hline \multicolumn{9}{|c|}{$\begin{array}{l}\text {.varsoc GrowsSpct Y_t InGt I } \\
\text { Selection-order criteria (lutstat) } \\
\text { Sample:2008-2018 }\end{array}$} \\
\hline Lag & LL & LR & $\mathrm{df}$ & $\mathrm{p}$ & FPE & AIC & HQIC & SBIC \\
\hline 1 & -350.152 & . & 25 & & $4.1 \mathrm{e}+23$ & 54.0201 & 53.45 & 54.9244 \\
\hline 2 & 471.404 & 1643.1 & 25 & 0.000 & $1.7 \mathrm{e}-37^{*}$ & -90.8083 & -91.9483 & -88.9996 \\
\hline 3 & 918.407 & $894.01 *$ & 25 & 0.000 & & $-167.536^{*}$ & $-169.246^{*}$ & $-164.823 *$ \\
\hline 4 & 832.465 & -171.88 & 25 & & & -147.365 & -140.654 & -143.748 \\
\hline
\end{tabular}

Note: Endogenous: GrowsSPCT, Y_t, InGt, InCt, InPt. Exogenous:_cons

Source: Own calculations.

Table 10. ARDL_cointegration test_Lag_Order 2.

.varsoc GrowsSpct Y_t InGt InCt, InPt, maxlag (2) noconstant lutstats separator(5>).)

Selection-order criteria (lutstat)

Sample:2006-2018 Number of obs $=13$

\begin{tabular}{|l|l|l|l|l|l|l|l|l|}
\hline Lag & LL & LR & df & p & FPE & AIC & HQIC & SBIC \\
\hline 0 & -428.541 &. & 25 &. & $1.7 \mathrm{e}+24$ & 55.5862 & 55.3629 & 56.6726 \\
\hline 1 & 63.2773 & $983.64 *$ & 25 & 0.000 & $1.1 \mathrm{e}+06$ & $-16.232^{*}$ & $-16.6787 *$ & $-14.0592 *$ \\
\hline
\end{tabular}

(See the pioneering work of Engle and Granger (1987), Johansen (1991), Phillips (1991), Phillips and Hansen (1990) and Phillips and Loretan (1991). 
Note: Endogenous: GrowsSPCT, Y_t, InGt, InCt, InPt. Exogenous:_cons

Source: Own calculations.

Table 11. ARDL Cointegration test

\begin{tabular}{|c|c|c|c|c|c|c|c|c|}
\hline \multicolumn{3}{|c|}{$\begin{array}{l}\text {.varsoc GrowsSpct Y_t InGt } \\
\text { Selection-order criteria (lutstat) } \\
\text { Sample:2009-2018 } \\
\end{array}$} & \multicolumn{6}{|c|}{ Number of obs $=10$} \\
\hline Lag & LL & LR & $\mathrm{df}$ & $\mathrm{p}$ & FPE & AIC & HQIC & SBIC \\
\hline 0 & -259.482 & & & & $9.1 e+17$ & 40.545 & 40.545 & 40.545 \\
\hline 1 & -220.39 & 78.185 & 16 & 0.000 & $1.3 e+16$ & 35.9265 & 35.3954 & 36.4106 \\
\hline 2 & 308.801 & 1058.4 & 16 & 0.000 & $2.3 \mathrm{e}-27^{*}$ & -66.7117 & -67.7738 & -65.7434 \\
\hline 3 & 1028.53 & $1439.5^{*}$ & 16 & 0.000 & - & $-207.458 *$ & $-209.051 *$ & $-206.005^{*}$ \\
\hline 4 & 1008.76 & -39.55 & 16 & & - & -200.303 & -202.303 & -198.366 \\
\hline 5 & 1009.5 & 1.4927 & 16 & 1.000 & - & -197.252 & -197.252 & -194.831 \\
\hline
\end{tabular}

Note: Endogenous: GrowsSPCT, Y_t, InGt, InCt. Exogenous:_cons

Source: Own calculations.

Table 12. Summary data of ARDL_lagl test

\begin{tabular}{|l|l|l|l|}
\hline LAG & Df & p-value & AIC \\
\hline Lag_1 & 16 & .000 & 50.9458 \\
\hline Lag_2 & 16 & .000 & $50.9397 *$ \\
\hline
\end{tabular}

Source: Own calculations. Endogenous: GrowsSPCT, Y_t, InGt, InCt. Exogenous:_cons Source: Own calculations.

To further check the results Johansen Tests for Cointegration (Johansen, 1995) or the maximum possibility test were also performed as presented in Tables 13 and 14 . This test assumes that all variables must be endogenous.

From the results, we see that Eigenvlaue and trace statistics have a cointegration relationship between the variables. Granger's analysis of causality with 14 observations was also examined.

The results of the Granger Causality test are presented in Table 10 (Chi square statistics with P-values in parentheses), both models offer the same conclusion regarding the dynamic relationship between government consumption and family consumption but also the relationship with all other variables.

The results showed that there is a positive relationship that goes from family consumption to government consumption, savings, per capita income.

But this positive scientific relationship exists in the long term, but not in the short term. 
Dynamic Relationship Between Government Spending, Family Consumption, and Savings: Evidence from Southeast Europe

534

Table 13. Johansen Tests for Cointegration

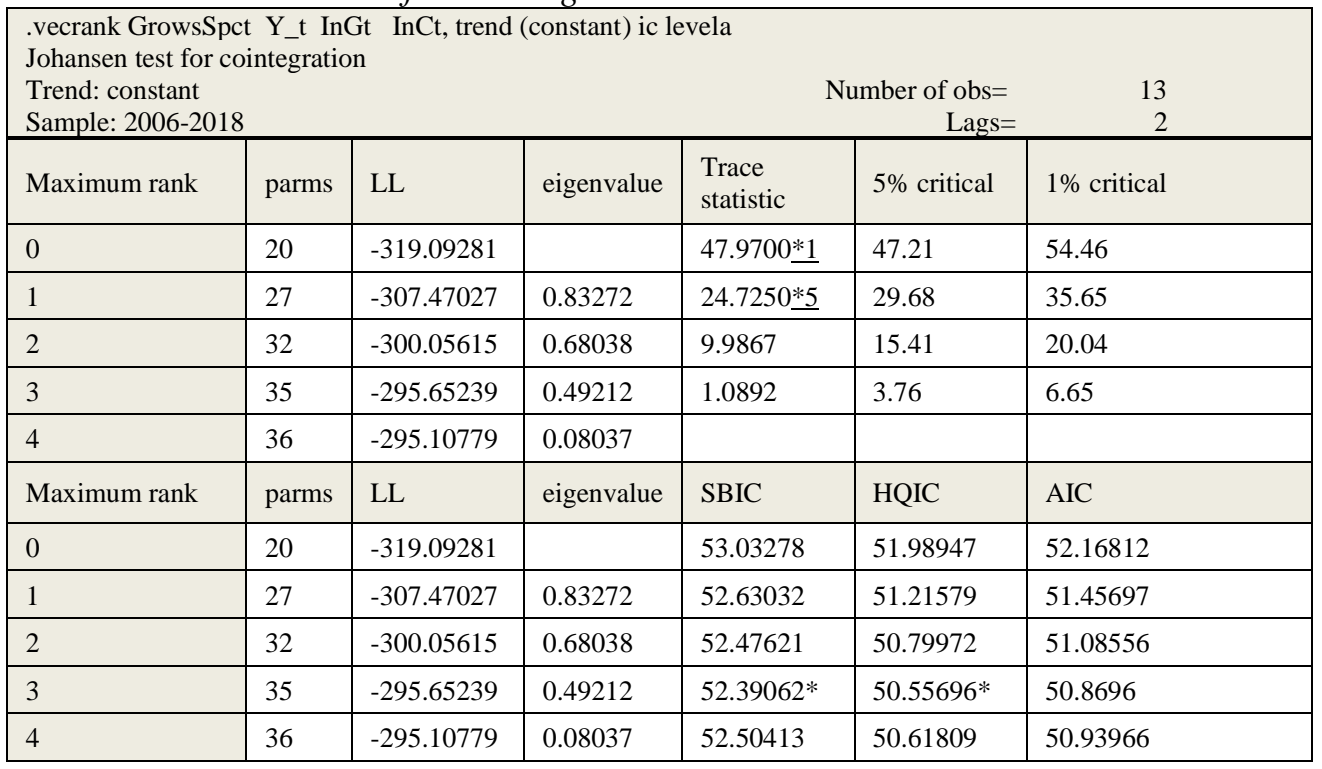

Source: Worked by the author.

Table 14. Data summary of Johansen Tests for Cointegration

\begin{tabular}{|l|l|l|l|}
\hline & Eigenvalue & SBIC & AIC \\
\hline 0 & - & 50.03728 & 52.16812 \\
\hline 1 & 0.83272 & 52.63032 & 51.45698 \\
\hline 2 & 0.68038 & 52.47621 & 51.08556 \\
\hline 3 & 0.49212 & $52.39062^{*}$ & 50.8696 \\
\hline 4 & 0.08037 & 52.50413 & 50.93966 \\
\hline
\end{tabular}

Note: $r$ : Indicates the number of cointegrating vectors. The Akaike information criterion was used to select the number of lags required in the cointegrating test. *Indicates the rejection of the null hypothesis of no-cointegration at the $5 \%$ level.

Source: Worked by the author.

Table 15. Results of Granger causality tests(sample 2005-2018)

\begin{tabular}{|c|c|c|c|}
\hline \multirow[t]{2}{*}{$\begin{array}{l}\text { Model /Dependen } \\
\text { Variable }\end{array}$} & $\begin{array}{l}\text { Long-run causality } \\
\mathrm{ECT}_{\mathrm{t}-1}=0\end{array}$ & \multirow[t]{2}{*}{$\begin{array}{l}\text { Model/ Dependen } \\
\text { Variable }\end{array}$} & $\begin{array}{l}\text { Long-run causality } \\
\mathrm{ECT}_{\mathrm{t}-1}=0\end{array}$ \\
\hline & Model 1-InCt & & Model 2- $\mathrm{InG}_{\mathrm{t}}$ \\
\hline $\begin{array}{l}\Delta \text { Model }_{1-I n C t} \\
\Delta \mathrm{InGS}_{\mathrm{t}} \\
\Delta \mathrm{InY}_{\mathrm{t}} \\
\Delta \mathrm{InG}_{\mathrm{t}} \\
\Delta \mathrm{InC}_{\mathrm{t}}\end{array}$ & $\begin{array}{l}0.882 *(0.0003) \\
0.957 *(0.0000) \\
0.960 *(0.0000) \\
0.933 *(0.0000)\end{array}$ & $\begin{array}{l}\text { Model 2- } \mathrm{InG}_{\mathrm{t}} \\
\Delta \mathrm{InGS}_{\mathrm{t}} \\
\Delta \mathrm{InPT}_{\mathrm{t}} \\
\Delta \mathrm{InG}_{\mathrm{t}} \\
\Delta \mathrm{InCS} \\
\Delta \mathrm{InY}_{\mathrm{t}}\end{array}$ & $\begin{array}{l}0.8841 *(0.0014) \\
0.9961 *(0.0000) \\
0.9608 *(0.0000) \\
0.9367 *(0.0001) \\
0.9579 *(0.0000)\end{array}$ \\
\hline
\end{tabular}

Note: C: Real per capita household final consumption, Y: Real per capita GDP, G: Real per capita government final consumption. Statistics for Short-run causality are Chi-square statistics with $P$ values in parentheses. The asterisk *denotes statistical significance at the $5 \%$ levels; Source: Worked by the author. 
The following Granger Causality results for model 1, in Table 15, show how, during the period 2005-2018, GrowsSpct, Y_t, InGt and InCt, in a long term lag _1, have a significant increase. At the GrowsSpct test $\mathrm{F}$ of statistical significance $=16.93368$, while $\mathrm{p}$ value $=.0003$, then $\mathrm{Yt}(\mathrm{F}=50.77761, \mathrm{p}$ value $=.000)$, InGt $(\mathrm{F}=$ $54.74031, \mathrm{p}$ value $=.000)$ and $\operatorname{InCt}(\mathrm{F}=31.39302, \mathrm{p}$ value $=.000)$.

Even model 2 in Table 16 shows approximately the same results in the long term but not more than lag_1.

Table 16. Model 1- Granger Causality test

\begin{tabular}{|c|c|c|c|c|c|}
\hline \multicolumn{6}{|c|}{.var GrowsSpct Y_t, InGt, InCt, lags (1/) smal dfk } \\
\hline \multicolumn{6}{|c|}{ Vector autoregresion } \\
\hline \multicolumn{6}{|c|}{ Sample:2005-2008 } \\
\hline & \multicolumn{3}{|c|}{$\mathrm{AIC} \quad=50.74359$} \\
\hline \multicolumn{3}{|c|}{ FPE $\quad=1.46 \mathrm{e}+17$} & HQIC & $=50.65902$ & \\
\hline \multicolumn{6}{|c|}{ Det $($ Sigma_ml $)=7.36 \mathrm{e}+15$} \\
\hline Equation & Parms & RMSE & R-sq & \begin{tabular}{|l|l|} 
& $\mathrm{F}$ \\
\end{tabular} & $\mathrm{P}>\mathrm{F}$ \\
\hline GrowthSpct & 5 & 75.8271 & 0.8827 & 16.93368 & 0.0003 \\
\hline Y_t & 5 & 124.041 & 0.9576 & 50.77761 & 0.0000 \\
\hline InGt & 5 & 722.979 & 0.9605 & 54.74031 & 0.0000 \\
\hline $\mathrm{InCt}$ & 5 & 95.3729 & 0.9331 & 31.39302 & 0.0000 \\
\hline
\end{tabular}

Source: Worked by the author.

Table 17. Model 2 - Granger Causality test

\begin{tabular}{|c|c|c|c|c|c|}
\hline \multicolumn{6}{|c|}{$\begin{array}{l}. \text { var GrowsSpct Y_t, InGt, InCt, Y_t, lags }(1 / 1) \text { small dfk lutstats vsquish cformat } \\
>(\% 09, \mathrm{Ogc}) \text { pformat }(\% 05, \mathrm{Ogc}), \text { sformat }(\% 0.80 \mathrm{gc})\end{array}$} \\
\hline \multicolumn{6}{|c|}{ Vector autoregresion } \\
\hline \multicolumn{3}{|c|}{ Sample:2005-2008 } & \multicolumn{3}{|c|}{ no. of obs. $=14$} \\
\hline Loglikelihod & -455.0331 & & AIC & $=54.38677$ & \\
\hline FPE & $1.44 \mathrm{e}+24$ & & HQIC & $=54.28114$ & \\
\hline \multicolumn{3}{|c|}{ Det $($ Sigma_ml $)=7.36 \mathrm{e}+15$} & SBIC & $=55.52795$ & \\
\hline Equation & Parms & RMSE & R-sq & \begin{tabular}{l|l|} 
& $F$ \\
\end{tabular} & $\mathrm{P}>\mathrm{F}$ \\
\hline GrowthSpct & 6 & 79.9539 & 0.8841 & 16.93368 & 0.0003 \\
\hline $\mathrm{InPt}$ & 6 & 34244.39 & 0.9961 & 50.77761 & 0.0000 \\
\hline InGt & 6 & 763.902 & 0.9608 & 54.74031 & 0.0000 \\
\hline $\mathrm{InCt}$ & 6 & 101.515 & 0.9367 & 31.39302 & 0.0000 \\
\hline InY_t & 6 & 130.976 & 0.9579 & & \\
\hline
\end{tabular}

Source: Worked by the author.

\section{Discussion}

The study problem focuses on the research question, "Are there positive short-term or long-term relationships between final consumption and government spending?". The use of different econometric approaches and strategies in the context of theories has led to different conclusions.

The results of our study are similar to those of Keho (2019, p. 197), which also show the dynamic relationship between government spending and family consumption in Cote d'Ivoire covering the period 1970-2016. "The results reveal a long run 
relationship between household consumption, real gross domestic product and government consumption. In the long run, private consumption and per capita income have positive effects on government consumption. However, in the short run there is no causal relationship between the variables" (Keho, 2019).

Also Karras (1994) quoted by Nazgul Agibaeva (2015, p. 7), has analyzed the relationship between private consumption and public consumption and its empirical results show that, in general, private consumption and government consumption are described as complementary in the sense that an increase in one consumption raises the marginal utility in the other consumption and all this depends on the size of the government. Baxter and King (1993, p. 315) emphasize, "Our findings reveal that in addition to the presence of complementarity, productivity - even if minimalincreases the likelihood of generating a positive consumption response". Linnemann and Schabert (2003, p. 911) analyze, the cyclical effects of fiscal policy shocks and come to the conclusion that "Price stickiness has the consequence that a rise in government demand affects labor demand, while at the same time the usual wealth effect boosts labor supply".

Gali, Lupez-Salido and Valles (2007, p. 260), in their study emphasize that "The com-bined effect of a higher real wage and higher employment raises current laborincome and hence stimulates the consumption of rule-of-thumb households". It is worth noting that the authors, Mir and Mansur (2012), during the testing of the Granger causality model in their study they do not find long-term causal relationships between government consumption and family consumption. Their study is equivalent to the Barro-Ricardian study of government spending that family consumption has nothing to do with the decision of government spending in the long run. These studies are contrary to our study, where we find a positive dynamic relationship between family consumption and long-term government spending (however not more than a year lag).

So our results consider private consumption and government consumption as complementary to each other. It should be noted that the results of our study may differ from some studies by the fact that the model used is modified for national savings (GSt) and total population (PTt) variables, also the rate of economic development of countries in the sample is different in the countries compared. The limiting factor may be a not very large sample (5 countries x 15 years $=75$ observations), compared to other countries that have included large samples. Interest in such a study is the ongoing debate in European countries that have been hit by the recession in 2008.

Should government spending increase related to GDP? In these cases, it has been difficult to determine the fiscal policies that stimulate the economy when it is not known whether there will be an increase in GDP. However, fiscal policies stimulate at least private consumption. Since family consumption accounts for the largest share of GDP, then increase in government spending along with investment should 
be followed at a close percentage. Some authors find that a government spending leads to a significant increase in consumption, while investment either falls or does not respond significantly (Galí, López-Salido and Vallés, 2004, p.33).

As a constraint of this study, among other things, we consider the non-inclusion of the variables of taxes, financial market and labor market, which we consider as factors that affect consumer behavior to consume or save.

\section{Conclusions}

The study provides answers to the research question, the dynamic relationship between family consumption, government spending, per capita income and gross savings in Southeast Europe, part of which is Kosovo. The correlation between variables in all cases is statistically significant and positive, except for the population which is statistically significant but negative. Even at the ARDL and Johansen approach, we found a positive long-term relationship between family consumption, government spending, per capita income and savings, in the case of using lag1 and lag2 time delays. Long-term relationships exist especially when government consumption is used as dependent or the other case when government spending is used as dependent. In the long run, the population has a negative effect on family consumption, government consumption, savings and per capita income.

The Granger causality test applied to error correction models suggests that all variables except the population that has been eliminated from the model cause a positive effect on each other, but in the long run with a lag1. The long-term positive effect of family consumption and government consumption may be the result of high consumption by the population and the government, which have generally increased taxes by increasing state revenues. But in the short term there is no causality between variables. It would be very important in future research to investigate the relationship between family consumption and government spending at the local and central level that would help employment and public investment policies shift to local levels with a weak supply chain.

\section{References:}

AECOM. 2016. Republic of Kosovo, Central Government Repeat Public Expenditure and Financial Accountability Assessment (PEFA). https://www.pefa.org/sites/default/files/.

Baxter, M., King, G. 1993. Fiscal Policy in General Equilibrium. American Economic Review, 83, 315-334. https://www.scirp.org/(S(i43dyn45teexjx455qlt3d2q).

Basu, P., Kollmann, R. 2013. Productive Government Purchases and the

Real Exchange Rate. The Manchester School, 81(4), 461-469.

https://ssrn.com/abstract=2282322.

Bouakez, H., Rebei, N. 2007. Why Does Private Consumption Rise after a

Government Spending Shock? Canadian Journal of Economics, 40, 954-979.

10.1111/j.1365-2966.2007.00438.x.

Bebezuk, R.N., Musalem, A.R. 2006. Pensions and saving: New International Panel 
Data Evidence. CEF, Centro para la Estabilidad Financiera, 1-19.

https://www.researchgate.net/signup.SignUp.html.

Barro, R.J. 1981. Output effects of government purchases. Journal of Political

Economy 89(6), 1086-1121. doi:10.1086/261024.

Bank of Canada Banque du Canada. Working Paper 2003-43. Document de travail 2003-43.

Why Does Private Consumption Rise Aftera Government Spending Shock? By Hafedh

Bouakez and Nooman Rebei, 1-33.

http://citeseerx.ist.psu.edu/viewdoc/download?doi=10.1.1.202.1714\&rep=rep1\&.

Chapsa, X., Tabakis, N., Athanasenas, L.A. 2018. Investigating the Catching-Up

Hypothesis Using Panel Unit Root Tests: Evidence from the PIIGS. European

Research Studies Journal, 21(1), 250-271.

Coenen, G., Straub, R. 2005. Does Government Spending Crowd in Private

Consumption: Theory and Empirical Evidence from the Euro Area. International

Finance, 8, 435-470.

Blanchard, O.J., Perotti, R. 2002. An Empirical Characterization of the Dynamic Effects of Changes in Government Spending and Taxes on Output. Quarterly Journal of Economics, 117, 1329-1368. https://doi.org/10.1162/003355302320935043.

Granger, C.W.J. 1988. Some Recent Development in a Concept of Causality. Journal of Econometrics, 39, 199-211.

Jordi Galí, J. David López-Salido, Javier Vallés. 2007. Understanding the Effects of Government Spending on Consumption, Journal of the European Economic

Association, Volume 5, Issue 1, 227-270.

https://doi.org/10.1162/JEEA.2007.5.1.227

Johansen, S. 1995. Likelihood based inferences in cointegrated vector autoregressive models. Oxford, Oxford University Press.

Ercolani, V. 2007. The relation between private consumption and government spending: theory and investment in Iran. Economic Research, (19), 235-262. https://www.sid.ir/en/Journal/.

Karras, G. 1994. Government Spending and Private Consumption: Some International Evidence. Journal of Money, Credit and Banking, 26(1), 9-22. doi:10.2307/2078031 https://www.jstor.org/stable/2078031?seq=1.

Karami, A. 2008. Impact of liquidity on growth rate of national savings. Journal of Money, Credit and Banking, Business Research, 46, 254-231.

Keynes, J.M. 1936. The General Theory of Employment, Interest and Money. Macmillan, London, United Kingdom. https://www.files.ethz.ch/isn/125515/1366_.

Linnemann, L., Schabert, A. 2003. Fiscal Policy in the New Neoclassical Synthesis. Journal of Money, Credit and Banking, 35, (6), 911-29. https://econpapers.repec.org/article/mcbjmoncb.

Linnemann, L. 2005. The effect of government spending on private consumption: a puzzle? Journal of Money, Credit and Banking, 38(7), 1715-1735.

Mojtahed, A., Karami, A. 2003. Evaluation of the determinants of the national savings behavior in Iranian economy. The Quarterly Journal of Commerce, (27), 1-28.

Mousavi, N., Monjaze, M. 2014. The Impact of growth rate of real GDP per capita on the savings rate in Iran and some developing and developed selected countries. International Journal of Scientific Management and Development, 2(9), 425-443.

Mahmud, Mir., Ahmed, Mansur. 2012. Government expenditure and household consumption in Bangladesh through the lens of economic theories: An Empirical Assessment. MPRA. https://mpra.ub.uni-muenchen.de/36035/.

Ministry of Finance. 2016. Medium Term Expenditure Framework 
2017-2019. 29-30. Available at:

https://mf.rks-gov.net/desk/inc/media/B633ADAA-875A-480B-87AF-DE.

Nazgul, Agibaeva. 2015. Government Spending and Private Consumption: Crowding In or Crowding Out? Evidence from Norway. Department of Economics, University of

Oslo. Available at: http://www.duo.uio.no/

Pesaran, H., Shin, Y. 1999. An autoregressive distributed lag modelling approach to cointegration analysis. In S. Strom (Ed.), Econometrics and economic theory in the 20th century: The Ragnar Frisch centennial symposium. Cambridge, Cambridge University Press.

Ravn, M.O., Schmitt, G., Stephanie, U.M. 2007. Explaining the Effects of Government Spending Shocks on Consumption and the Real Exchange Rate. NBER Working Paper, no. 13328. https://www.nber.org/papers/w13328.pdf.

Bhattacharya, R., Mukherjee, S. 2010. Private sector consumption and government consumption and debt in advanced economies: An empirical study (Working Paper WP/10/264). Washington, DC, International Monetary Fund.

Ramey, V., Shapiro, M. 1998. Costly Capital Reallocation and the Effect of Government Spending. Carnegie-Rochester Conference Series on Public Policy, 48, 145-194. https://www.sciencedirect.com/science/article/abs/pii/S0167223198000207.

Sadi, M. 2006. Effects of macroeconomic policies in mobilizing domestic resources for investment in Iran. Economic Research. (19), 235-262. https://www.sid.ir/en/Journal/.

Keho, Y. 2019. Dynamic Relationship between Government Spending and Private Consumption: Evidence from Cote d'Ivoire. International Journal of Economics and Financial Issues, 9(1), 197-202. DOI: https://doi.org/10.32479/ijefi.7433.

\section{Source online}

http://documents.worldbank.org/curated/en/992481566310183572/Fiscal-Rules-for-theWestern-Balkans. http://pubdocs.worldbank.org/en/159411478200087521/Albania-SnapshotOct2016FINAL.pdf. https://www.worldbank.org/en/region/eca/brief/country-program-snapshots. http://web.worldbank.org/archive/website01352/WEB/IMAGES/KOSOVO-4.PDF https://mf.rks-gov.net https://davegile.blogspot.com/2013/06/ardl-models-part-ii-bounds-tests.html). https://davegile.blogspot.com/2013/06/ardl-models-part-ii-bounds-tests.html. 